\title{
Electrical transport in ethyl cellulose-chloranil system
}

\author{
P K KHARE*, R K PANDEY ${ }^{\dagger}$ and P L JAIN \\ Department of Postgraduate Studies and Research in Physics and Electronics, Rani Durgavati University, \\ Jabalpur 482 001, India \\ ${ }^{\dagger}$ Department of Physics, Dr H S Gour University, Sagar 470 002, India \\ ${ }^{\ddagger}$ Government Postgraduate College, Damoh 470 661, India \\ MS received 11 January 2000; revised 24 May 2000
}

\begin{abstract}
The charge-transport behaviour in pure and chloranil (Chl) doped ethyl cellulose (EC) system has been studied by measuring the dependence of current on field, temperature, electrode material and dopant concentration. The role of doping molecular concentration in the polymer matrix and modification in the conduction characteristics are studied. Lowering of the activation energy due to doping was observed. The current was found to increase with an increase in the chloranil concentration. An explanation for this has been attempted on the basis of formation of molecular aggregates between chloranil molecules and ethoxy groups of ethyl cellulose. It is suggested that chloranil occupies interstitial positions between the polymer chains and assists in carrier transportation by reducing the hopping barriers. The current-voltage characteristics of different samples are analyzed using space charge limited current theory and quantitative information about the transport parameters is derived. The values of effective drift mobility and trapped charge carrier concentration which result in the build up of space charge have been calculated.
\end{abstract}

Keywords. Electrical transport; ethyl cellulose; chloranil; charge transfer complex; molecular aggregates; Richardson-Schottky; Poole-Frenkel; space charge limited current.

\section{Introduction}

The transport of electric charge across the surfaces of insulators is a topic of considerable technical interest, but one in which the mechanisms involved are poorly understood in general. The elucidation of the charge injection and carrier migration processes will become essential for the future use of these materials (Shrivastava et al 1979; Khare and Srivastava 1992). The study of electrical conduction is of considerable significance from two major points of view-firstly, for its own sake, because charge transfer characteristics are of fundamental interest and secondly for the information studies can provide on the nature of electrical contacts, which may have a great influence on the measured electrical properties. The charge storage property of polymers can be considerably modified with the help of suitable dopants (Khare et al 1998a). The broadest method that can be applied to enhance the conductivity of polymeric material is the formation of molecular aggregates or charge-transfer (CT) complexes. The electrical conduction behaviour of a number of polymer CT complexes and molecular aggregates has been investigated recently (Khare and Singh 1994; LaxmiNarayana et al 1994; Khare and Chandok 1995a; Khare and Jain 1997; Upadhyay et al 1998), yet the mechanism

*Author for correspondence of conduction and the role of impurity in these systems is not known to any degrees of certainty. The carrier mobility can be greatly influenced by impregnating the polymers with suitable dopants (Khare and Singh 1994; Khare et al 1997a, 1998b).

The present paper describes the electrical transport in the ethyl cellulose (EC)-chloranil (Chl) system observing the variation of current under different conditions of poling temperatures, electrode materials, thicknesses and Chl concentrations.

\section{Experimental}

Ethyl cellulose (EC) and an electron acceptor chromatographically purified chloranil (Chl) doped EC films were deposited onto cleaned vacuum aluminium coated glass plates by isothermal immersion of the substrates into a fresh solution of well-stirred mixtures of $3.0 \mathrm{~g}$ of EC in $30 \mathrm{ml}$ of chloroform at room temperature for $10 \mathrm{~min}$. Using the same technique, we also prepared $\mathrm{Chl}$ doped EC films under identical conditions from solutions of well-stirred mixtures of $3.0 \mathrm{~g}$ of EC and Chl in chloroform. The doping concentration was changed by varying the amount of $\mathrm{Chl}$ added to the solvent such that the total volume of the solvent and the Chl was always $30 \mathrm{ml}$. Although samples with different dopant concentrations were investigated, only the results for two types of 
samples are reported here. The samples doped to the lower concentration of $\mathrm{Chl}(1 \%)$ and higher concentration of Chl (4\%) were designated as $\mathrm{P}_{1}$ and $\mathrm{P}_{2}$, respectively. The further increase in the dopant concentration does not result in increase of the current. The films were dried and determined to be $\approx 20 \mu \mathrm{m}$ thick, by measurement of their capacitance. After the film deposition the glass slide was wrapped in an aluminium mask. When exposed to aluminium/silver/copper in vacuum, the metal was deposited on the film. Thus, a sample in the sandwich structure was obtained. The activation energies for the undoped samples $(\approx 0.63 \mathrm{eV}$ in the lower temperature region and $0.52 \mathrm{eV}$ in the higher temperature region) were found to be decreased as a result of $\mathrm{Chl}$ doping (to $0.58 \mathrm{eV} ; 0.43 \mathrm{eV}$ and $0.55 \mathrm{eV} ; 0.40 \mathrm{eV}$ for lower and higher $\mathrm{Chl}$ concentrations, respectively). A d.c. power supply EC-4800 and a Keithley 600B electrometer were used to apply the desired electric field and record the current. Measurements were made at thermostatically controlled temperatures in the range 313-363 K.

\section{Results and discussion}

Figure 1 shows the current-voltage $(I-V)$ characteristics of undoped and chloranil (Chl) doped ethyl cellulose (EC) samples. At the lower concentration, the current was found to be lower. As the dopant concentration was increased, the current was found to increase. The effect of electrode materials on $I-V$ characteristics of undoped and Chl doped EC matrix (all taken at $323 \mathrm{~K}$ ) has also been placed in the same figure. The magnitude of the current was found to be higher in dissimilar electrode combinations than similar electrode system. The $I-V$ curves show two regions of conduction, i.e. ohmic conduction at lower fields $(<20 \mathrm{kV} / \mathrm{cm})$ and a non-ohmic conduction at higher fields ( $>20 \mathrm{kV} / \mathrm{cm}$ ) for the undoped and doped samples. Figure 2 shows the $I-V$ characteristics for undoped and doped films of different thicknesses. Curves 1 to 5 correspond to thicknesses $30,25,20,15$ and $10 \mu \mathrm{m}$, respectively. It is seen from these curves that there are distinctly two regions. Further, the transition voltage $\left(V_{\mathrm{tr}}\right)$ at which the non-ohmic behaviour starts appearing is seen to depend on the thickness of the film.

The above results indicate that with the increase of temperature, the probability of thermal ionization of the trapping centres increases thus causing a shift in the quasi-Fermi level, which gives rise to a lowering of the barrier across which electrons have to be transported and the conduction becomes more or less ohmic. At lower fields, the injection of carriers from contact is less and the initial current is governed by the intrinsic free carriers in the materials. The current will be ohmic until the injected free carrier density becomes comparable with the thermally created carrier density. However, at sufficiently higher fields, the carriers are mainly due to injected space charges (Khare and Chandok 1995b; Khare et al 1998a).
The high-field electronic conduction mechanism commonly discussed for various films are space-charge limited conduction (SCLC), Richardson-Schottky (R-S), Poole-Frenkel (P-F) emission and tunneling of carriers into or via traps (Lamb 1967; Simmons 1970). In order to establish the dominant mode of conduction in a particular material, one has to look into the detailed analysis of $I-V$ data of the material in terms of the theoretical considerations available for different types of processes.

The $I-V$ characteristics follow the relationship of the form $\log I$ vs (field) ${ }^{1 / 2}$ and thus predict either the Richardson-Schottky (R-S) limited mechanism from the electrodes or the bulk-limited Poole-Frenkel (P-F) effect. The latter effect is caused by thermal ionization of trapped electrons from donor-like centres in the presence of an external field. If one considers the RS or PF emission as the dominant mode of conduction then the current density I follows the relation (Lamb 1967; Simmons 1970)

$$
I=I_{0} \exp \left(\beta E^{1 / 2} / k T\right)
$$

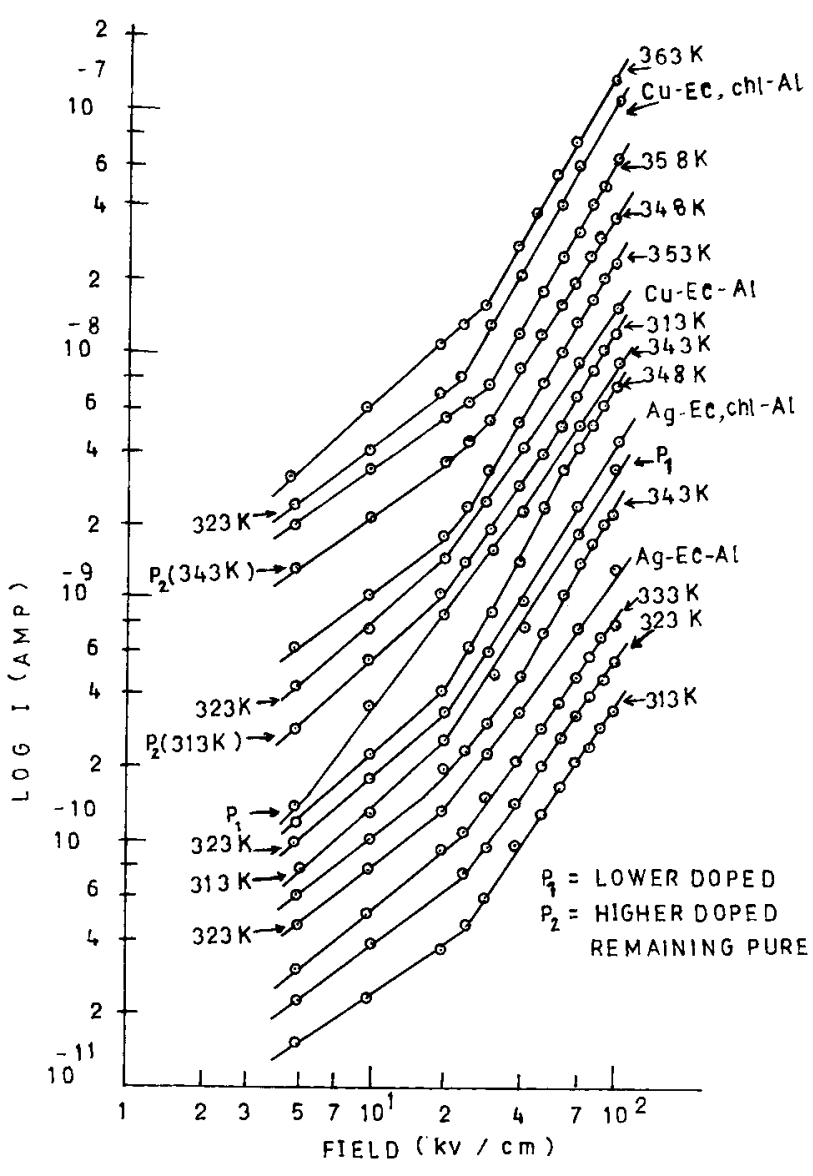

Figure 1. Current-voltage characteristics of pure and Chl doped ethyl cellulose films with similar and dissimilar electrode systems. 
where

$$
I_{0}=A T^{2} \exp (-\phi / k T) \quad \text { for RS, }
$$

and

$$
I_{0}=\sigma_{0} V / d \quad \text { for } \mathrm{PF},
$$

and the constants

$$
\beta_{\mathrm{RS}}=\left(e^{3} / 4 \pi \varepsilon \varepsilon_{0}\right)^{1 / 2}=1 / 2 \beta_{\mathrm{PF}},
$$

where $\varepsilon_{0}$ is the permittivity of vacuum, $A$ the Richardson's constant, $\sigma_{0}$ the low field conductivity and $E$ the applied field, i.e. $E=V / d$. Equation (1) suggests that the plot of $\log I$ vs $E^{1 / 2}$ should be a linear one having a slope of $\beta / k T$. The value of $\beta$ was estimated from such graphs. Table 1 gives the theoretical values of $\mathrm{R}-\mathrm{S}$ coefficients $\left(\beta_{\mathrm{RS}}\right)$ and $\mathrm{P}-\mathrm{F}$ coefficients $\left(\beta_{\mathrm{PF}}\right)$ along with the experimental values of $\beta$ for different pair of electrode combinations. Evidently the experimental values lie between the two theoretical values. The $\beta_{\exp }$ values complicate the interpretation of results in terms of either of these mechanisms. Jonscher and Ansari (1971) suggest that the basic difference between the two processes (one is electrode limited while the other is not) may be successfully utilized for selecting

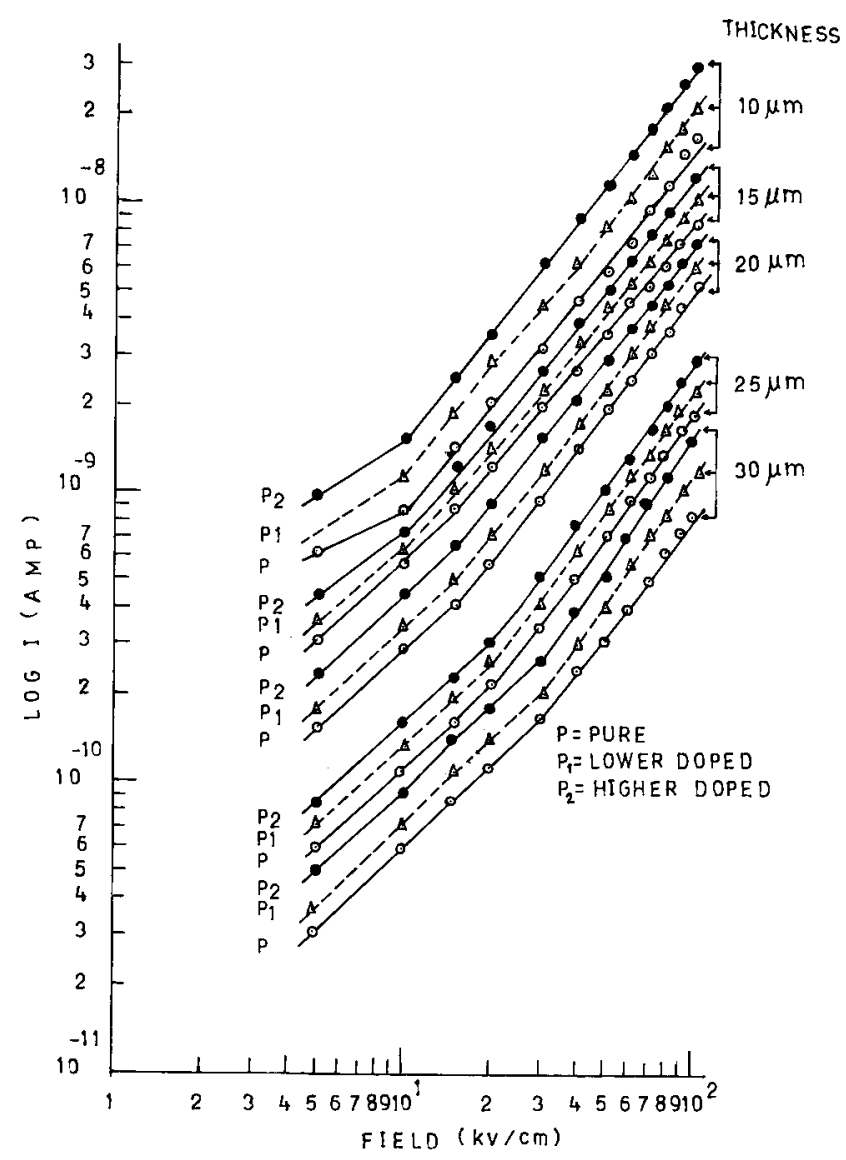

Figure 2. Current-voltage characteristics for undoped and Chl doped ethyl cellulose films of different thicknesses. the mechanism. Consequently, graphs are plotted between $\log I$ vs (field) ${ }^{1 / 2}$ (figure 3 ) using three different pairs of electrodes (i.e. $\mathrm{Al}-\mathrm{Al}, \mathrm{Al}-\mathrm{Cu}$ and $\mathrm{Al}-\mathrm{Ag}$ ). The plots trace a separate straight line. Further, by interchanging the + ve and - ve electrodes the current was found to be unchanged. The value of high frequency dielectric constant was calculated by using (2). It was found that on substituting the value of $\beta_{\mathrm{RS}}, \varepsilon>10$ and on substituting the value of $\beta_{\mathrm{PF}}, \varepsilon>17$. However, as the reported value for EC is $\approx 3.00$ (Mark et al 1965); such a large discrepancy in the obtained and reported values of $\varepsilon$ ruled out the possibility of RS or PF mechanisms governing the high field currents.

In the present case, in the high field conduction region, the $I-V$ characteristics have a slope of $>2$ and we suggest that the region is governed by space-charge-limited currents (SCLC). This is because, when the $I-V$ data are analyzed with the mathematical details available for SCL currents, a correspondence between theory and experimental values is observed.

In a trap-free solid, the space charge limited current (SCLC) $I_{0}$ is given by (Lampert and Mark 1970)

$$
I_{0}=\left(10^{-13} \mu_{\mathrm{e}} \varepsilon A V^{2}\right) / d^{3},
$$

where $\mu_{\mathrm{e}}$ is the effective drift mobility of the carriers, $\varepsilon$ the relative dielectric constant of the solid, $A$ the area of the sample, $d$ the thickness of the sample and $V$ the applied voltage. In presence of shallow traps only a fraction of the carriers injected from the contact will be free, the remainder will be immobile within the traps. The ratio of free carriers $n$ to trapped ones $n_{\mathrm{t}}$ is constant and independent of the applied voltage. In this range, the current $I_{\mathrm{t}}$ is given by (Lampert and Mark 1970)

$$
\begin{aligned}
& I_{\mathrm{t}}=\theta I_{0}, \\
& \theta=\frac{n}{n_{\mathrm{t}}}=\left(\frac{N_{\mathrm{c}}}{N_{\mathrm{t}}}\right) \exp \left(\frac{-E_{\mathrm{t}}}{k T}\right),
\end{aligned}
$$

where $N_{\mathrm{c}}$ is the effective density of states in conduction band and $N_{\mathrm{t}}$ represents the concentration of shallow trapping levels which are all assumed to have equal energy so that they are situated $E_{\mathrm{t}} \mathrm{eV}$ below the bottom of conduction band.

Conduction in polymers is explained not on the basis of band theory but in terms of the existence of mobility edge. Charge carriers are free to move only when they possess a certain value of mobility. If the charge carrier is near the bottom of conduction band, it is in the delocalized state and requires no thermal activation. However, if it is in the localized trapped state, it requires thermal activation and mobility increases with the increase in temperature (Ranjeet Singh et al 1997). The mobility values obtained in the present polymer are well within the limits of measured 
Table 1. Theoretical values of Richardson-Schottky coefficient $\left(\beta_{\mathrm{RS}}\right)$ and Poole-Frenkel (P-F) coefficient alongwith the experimental values of $\beta$.

\begin{tabular}{|c|c|c|c|c|}
\hline \multirow[b]{2}{*}{$\begin{array}{l}\text { Electrode } \\
\text { systems }\end{array}$} & \multirow[b]{2}{*}{$\begin{array}{l}\text { Temp. } \\
(\mathrm{K})\end{array}$} & \multicolumn{2}{|c|}{ Theoretical } & \multirow[b]{2}{*}{$\begin{array}{l}\text { Experimental } \beta \text {-values } \\
\qquad 10^{-21} \mathrm{JM}^{1 / 2} \mathrm{~V}^{-1 / 2}\end{array}$} \\
\hline & & $\begin{array}{c}\beta_{\mathrm{RS}} \\
10^{-21} \mathrm{JM}^{1 / 2} \mathrm{~V}^{-1 / 2}\end{array}$ & $\begin{array}{c}\beta_{\mathrm{PF}} \\
10^{-21} \mathrm{JM}^{1 / 2} \mathrm{~V}^{-1 / 2}\end{array}$ & \\
\hline \multirow{7}{*}{$\mathrm{Al}-\mathrm{Al}$} & 313 & $4 \cdot 768$ & $9 \cdot 536$ & $6 \cdot 829$ \\
\hline & 323 & $6 \cdot 523$ & $13 \cdot 046$ & $9 \cdot 857$ \\
\hline & 333 & $7 \cdot 125$ & $14 \cdot 250$ & $10 \cdot 543$ \\
\hline & 343 & $8 \cdot 066$ & $16 \cdot 132$ & $12 \cdot 667$ \\
\hline & 353 & $8 \cdot 997$ & $17 \cdot 994$ & $13 \cdot 689$ \\
\hline & 363 & $9 \cdot 117$ & $18 \cdot 234$ & $14 \cdot 114$ \\
\hline & & $10^{-20} \mathrm{JM}^{1 / 2} \mathrm{~V}^{-1 / 2}$ & $10^{-20} \mathrm{JM}^{1 / 2} \mathrm{~V}^{-1 / 2}$ & $10^{-20} \mathrm{JM}^{1 / 2} \mathrm{~V}^{-1 / 2}$ \\
\hline \multirow{6}{*}{$\mathrm{Al}-\mathrm{Cu}$} & 313 & $6 \cdot 442$ & $12 \cdot 884$ & $9 \cdot 857$ \\
\hline & 323 & $7 \cdot 179$ & $14 \cdot 358$ & $10 \cdot 014$ \\
\hline & 333 & $7 \cdot 892$ & $15 \cdot 784$ & $11 \cdot 312$ \\
\hline & 343 & $8 \cdot 023$ & $16 \cdot 046$ & $12 \cdot 444$ \\
\hline & 353 & $8 \cdot 563$ & $17 \cdot 126$ & $12 \cdot 999$ \\
\hline & 363 & $9 \cdot 222$ & $18 \cdot 444$ & $13 \cdot 764$ \\
\hline \multirow{6}{*}{$\mathrm{Al}-\mathrm{Ag}$} & 313 & $7 \cdot 234$ & $14 \cdot 468$ & $10 \cdot 854$ \\
\hline & 323 & $7 \cdot 997$ & $15 \cdot 994$ & $11 \cdot 111$ \\
\hline & 333 & $8 \cdot 256$ & $16 \cdot 512$ & $12 \cdot 447$ \\
\hline & 343 & $9 \cdot 345$ & $18 \cdot 690$ & $14 \cdot 875$ \\
\hline & 353 & 9.957 & $19 \cdot 914$ & $15 \cdot 789$ \\
\hline & 363 & $10 \cdot 634$ & $21 \cdot 268$ & $17 \cdot 412$ \\
\hline
\end{tabular}

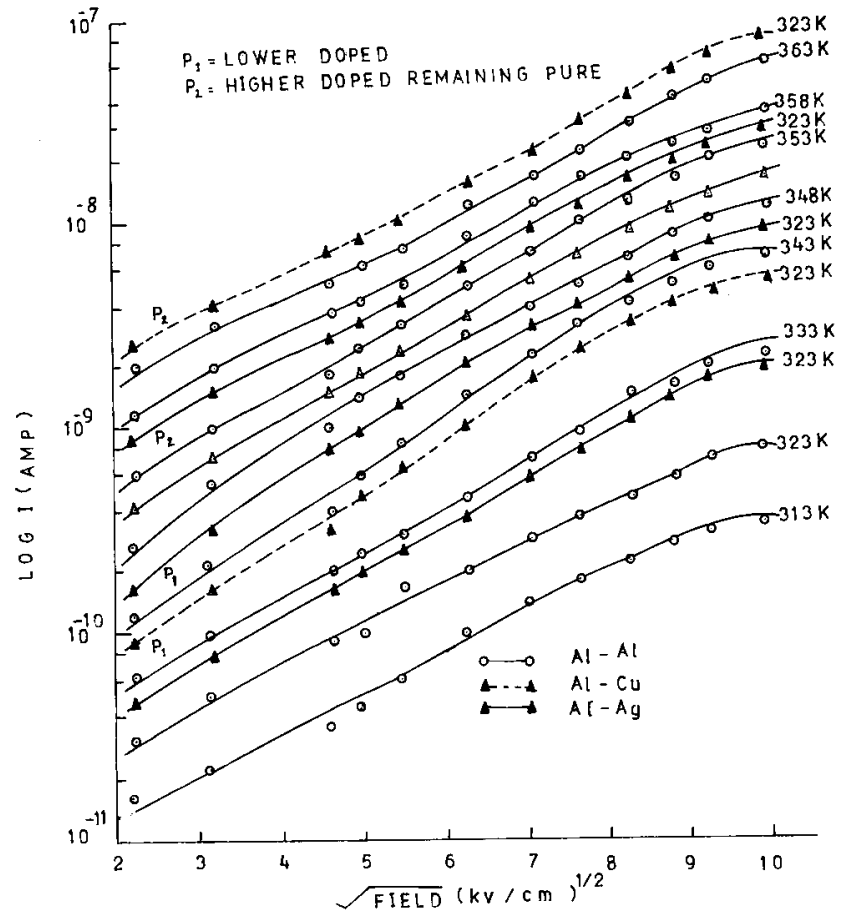

Figure 3. $\log I$ vs $\sqrt{\text { field }}$ plots.

mobilities in polymers (Wintle 1971; Davies and Lock 1973).

At higher fields (figure 1) the change of slope can be taken as an indication of deviation from Ohm's law into the space-charge limited current (SCLC) mechanism. This departure from Ohm's law in high field occurs as a result of active barrier perturbation even in the absence of thermal and chemical effects (Khare et al 1994). Space charge limited currents set in at that voltage $V_{\mathrm{t}}$ at which the intrinsic current equals the SCL current. Thus,

$$
\begin{aligned}
& n_{0} e \mu_{\mathrm{e}}\left(\frac{V_{\mathrm{t}}}{d}\right)=10^{-13} \mu_{\mathrm{e}} \varepsilon \theta\left(\frac{V_{\mathrm{t}}^{2}}{d^{3}}\right) \\
& V_{\mathrm{t}}=10^{13} n_{0} e d^{2}(\varepsilon \theta)^{-1},
\end{aligned}
$$

where $n_{0}$ is the concentration of free carriers before injection. Thus, the voltage at which deviation from ohmic behaviour takes place is a measure of the density of the normal volume-generated carriers.

The trap concentration $N_{\mathrm{t}}$ is defined by

$$
N_{\mathrm{t}}=\frac{2 \varepsilon \varepsilon_{0}}{e d^{2}} V_{\mathrm{TFL}},
$$

where $e$ is the electronic charge and $V_{\mathrm{TFL}}$ (trap-filled limit) is the upper limit of the voltage at which sufficient charge has been injected into the insulator to fill the traps. Using the experimental value of $V_{\mathrm{TFL}}, N_{\mathrm{t}}$ was calculated to be $8.765 \times 10^{14} \mathrm{~cm}^{-3}$. The values of $\mu_{\mathrm{e}}$ and $n_{\mathrm{t}}$ have been calculated for different thicknesses and are listed in table 3 . It is seen from table 3 that the charge carriers which result in formation of the space charge and hence limiting the high field conduction possess the average values of 
Table 2. Values of $\theta, \mu_{\mathrm{e}}, N_{\mathrm{t}}$ and $n_{0}$ for pure and chloranil doped ethyl cellulose.

\begin{tabular}{|c|c|c|c|c|c|c|c|c|c|c|c|c|}
\hline \multirow{3}{*}{$\begin{array}{l}\text { Temperature } \\
(\mathrm{K})\end{array}$} & \multicolumn{3}{|c|}{$\begin{array}{c}\theta \\
\left(\ldots \times 10^{-2}\right)\end{array}$} & \multicolumn{3}{|c|}{$\begin{array}{l}\text { Effective mobility }\left(\mu_{\mathrm{e}}\right) \\
\qquad\left(\ldots \times 10^{-9}\right) \\
\mathrm{m}^{2} \mathrm{v}^{-1} \mathrm{~s}^{-1}\end{array}$} & \multicolumn{3}{|c|}{$\left(\begin{array}{l}N_{\mathrm{t}} \\
\mathrm{cm}^{-3}\end{array}\right.$} & \multicolumn{3}{|c|}{$\begin{aligned} & n_{0} \\
&\left(\ldots \times 10^{10}\right) \\
& \mathrm{cm}^{-3}\end{aligned}$} \\
\hline & \multicolumn{3}{|c|}{ Sample } & \multicolumn{3}{|c|}{ Sample } & \multicolumn{3}{|c|}{ Sample } & \multicolumn{3}{|c|}{ Sample } \\
\hline & Pure & $P_{1}$ & $P_{2}$ & Pure & $P_{1}$ & $P_{2}$ & Pure & $P_{1}$ & $P_{2}$ & Pure & $P_{1}$ & $P_{2}$ \\
\hline 313 & $2 \cdot 637$ & $6 \cdot 425$ & $8 \cdot 875$ & 4.987 & $6 \cdot 678$ & $9 \cdot 875$ & $9 \cdot 935$ & $11 \cdot 864$ & $14 \cdot 743$ & $6 \cdot 567$ & $9 \cdot 87$ & $12 \cdot 654$ \\
\hline 323 & $3 \cdot 876$ & - & - & $5 \cdot 102$ & - & - & $9 \cdot 102$ & - & - & $7 \cdot 623$ & - & - \\
\hline 333 & $4 \cdot 678$ & - & - & $5 \cdot 654$ & - & - & $8 \cdot 654$ & - & - & $8 \cdot 118$ & - & - \\
\hline 343 & $6 \cdot 878$ & $9 \cdot 743$ & $12 \cdot 604$ & $7 \cdot 017$ & $10 \cdot 815$ & $11 \cdot 432$ & $8 \cdot 017$ & $10 \cdot 356$ & $13 \cdot 478$ & $9 \cdot 102$ & 11.456 & 13.578 \\
\hline 348 & $8 \cdot 957$ & - & - & $7 \cdot 654$ & - & - & $7 \cdot 764$ & - & - & $10 \cdot 111$ & - & - \\
\hline 353 & $9 \cdot 015$ & - & - & $8 \cdot 884$ & - & - & $6 \cdot 885$ & - & - & $11 \cdot 052$ & - & - \\
\hline 358 & $11 \cdot 143$ & - & - & $9 \cdot 667$ & - & - & $5 \cdot 888$ & - & - & 11.999 & - & - \\
\hline 363 & $12 \cdot 225$ & - & - & $10 \cdot 789$ & - & - & 4.985 & - & - & $12 \cdot 012$ & - & - \\
\hline
\end{tabular}

Table 3. Values of $\mu_{\mathrm{e}}$ and $N_{\mathrm{t}}$ with thickness (Al-Al electrode).

\begin{tabular}{lcc}
\hline $\begin{array}{l}\text { Thickness } \\
(\mu \mathrm{m})\end{array}$ & $\begin{array}{c}\mu_{\mathrm{e}} \\
\mathrm{cm}^{2} \times 10^{-1} \mathrm{~s}^{-1}\end{array}$ & $\begin{array}{c}N_{\mathrm{t}} \\
\left.\ldots \times 10^{14}\right) \\
\mathrm{cm}^{-3}\end{array}$ \\
\hline 10 & 3.967 & 12.478 \\
15 & 4.563 & 10.032 \\
20 & 4.987 & 9.987 \\
25 & 5.114 & 8.876 \\
30 & 5.635 & 8.012 \\
\hline
\end{tabular}

$\mu_{\mathrm{e}} \approx 4.854 \times 10^{-9} \mathrm{~cm}^{2} \mathrm{~V}^{-1} \mathrm{~s}^{-1}$ and $n_{\mathrm{t}} \approx 9.88 \times 10^{14} \mathrm{~cm}^{-3}$, respectively.

The equilibrium concentration of charge carriers in the conduction band can be obtained from the equation

$$
n_{0}=\frac{\varepsilon \varepsilon_{0} \theta}{q d^{2}} V_{\mathrm{tr}}
$$

where $V_{\text {tr }}$ is the voltage at which the transition from ohmic to square-law behaviour takes place. Again $\theta$ is the ratio of the free carrier density to the trapped carrier density and can be found from the ratio between the current at the start of the rise to that at the end of the rise in the squarelaw region. Using the value of $\theta, n_{0}$ was calculated from (8) and listed in table 2. It is to be noted that (i) $\log -\log$ plot of $I$ vs $d$ in the square-law region should be a straight line with slope -3 and $\log -\log$ plot of $V_{\text {tr }}$ vs $d$ should be a straight line with slope 2. Such plots were made (not shown) and the slopes of -2.92 and 1.93 , respectively were obtained which supports the possibility of space charge limited currents.

The space charge limited currents may also determine the transient behaviour such as a large burst of current immediately after the application of voltage followed by a steady decline in current on standing. In the present case the large currents obtained just after the application of voltage subsided to much smaller steady values after a certain length of time. The possible explanation is that the sudden application of voltage causes a cloud of carriers, i.e. a space charge, to be injected from the contact into the sample. This free charge gives rise to a large burst of current. If the space charge remained untrapped, the value of the transient current would continue as a steady current. However, one must take into account the effects of trap densities in the sample. The free charge forced into the sample settles the rate being determined by the capture cross section of traps for free carriers (Singh and Srivastava 1972).

In the present case, the slope of $I-V$ curves exceed the value 2 . Usually a slope of 2 indicates shallow trapping but this does not seem to exclude the possibility of deep traps in the present case. The attribution of the currents in high field region to SCL conduction is further supported by the following observation (i) EC being an amorphous material, would provide a large number of trapping centres and trapping of charge carriers in these trapping sites would result in the build-up of a space charge, (ii) the build-up of a space charge is also confirmed by our earlier thermally stimulated discharge current study (Khare et al 1996, 1997b).

The electrical conductivity for samples doped at lower concentration remains low even beyond glass transition temperature $T_{\mathrm{g}}$. It seems that chain motion is not effective in mobilizing the traps formed by dopant molecules and charge carriers released from mobilized traps, are again trapped in traps provided by dopant molecular sites. Thin polymer films are known to be a mixture of amorphous and crystalline regions (Chopra et al 1971). The conductivity behaviour of such films may be dominated by the properties of the amorphous regions (Kosaki et al 1971). The presence of amorphous regions give rise to localized states. Since there are many localized states, the release or excitation of the carriers in these states dominates the conduction process. Consequently, doping should not affect the conductivity too much (Marikhin et al 1965), 
unless the dopant is present in sufficient quantity to markedly affect the position of the Fermi level. Dopant molecules enter either the amorphous regions of the polymer or the defect regions like chain folds. If they are present in low concentration, they will give rise to additional molecular sites for trapping of charge carriers (Jonscher 1967). Such localized sites can be defined in molecular terms using the difference in ionization potential as an indication of trap depth. As the dopant concentration is increased, the molecules start bridging the gap separating the two localized states and lowering the potential barrier between them thereby facilitating the transfer of charge carriers (Kulshrestha and Srivastava 1980; Sinha et al 1981). A comparison of currents of pure and Chl doped EC reveals that an increase in current is observed with doping. Enhancement of current with an increase in Chl concentration may be explained on the basis of charge transfer complex (CTC) formation. Chloranil when doped in polymer, may reside at various sites. It may be substituted into the polymer chains or reside at the amorphous/crystalline boundaries and diffuse preferentially through the amorphous regions forming CTC, or it may exist in the form of molecular aggregates between the polymer chains. The CTC are supposed to create localized states of various depths which will lead to trapping sites distributed over a considerably wide energy range. The formation of CTC is considered to reduce the barrier between the trapping sites providing a conducting path through the polymer matrix and would result in the enhancement of its conductivity. Such a phenomena is supposed to decrease the activation energy of the carrier and increase its mobility towards the electrode during polarization. The increase in current with doping is supposed to lead to the accumulation of more and more positive charge in front of the cathode resulting in an increased strength at the polymer-electrode interface. In the process of polarization, the negative charge migrating under the influence of the charging field is supposed to accumulate in the vicinity of the anode due to presumably blocking polymer-metal contact and is immobilized during the cooling phase of the charging process (Khare and Singh 1994). In such cases, a considerable amount of charge is supposed to pass through the bulk of the sample and the observed current for doped samples should be sufficiently less than that in undoped samples by one or two orders in magnitude. Such observation goes against the possibility of CTC formation. The possibility of a new crystallite embedded in the amorphous mass cannot be precluded. Hence, it is reasonable to assume that $\mathrm{Chl}$ molecules occupy the interstitial positions between the polymer chains of amorphous phase and link these chains to some kind of bonds by a charge-exchange process between the Chl molecule and ethoxy group of EC. This process reduces the interstitial barriers and increase the transition probability of electrons hopping across these barriers. The interfacial barriers probably also decrease because of the presence of $\mathrm{Chl}$ at the interfaces. Thus, the whole structure of EC will be more open for charge transportation follow up impregnation with $\mathrm{Chl}$.

\section{References}

Chopra K L, Rastogi A C and Malhotra G L 1971 Thin Solid Films 24125

Davies W K and Lock P J 1973 J. Electrochem. Soc. 120266

Jonscher A K 1967 Thin Solid Films 1213

Jonscher A K and Ansari A A 1971 Philos. Mag. 23205

Khare P K and Srivastava A P 1992 Thin Solid Films 208233

Khare P K and Singh Ranjeet 1994 Polym. Int. 34407

Khare P K and Chandok R S 1995a Polym. Int. 3635

Khare P K and Chandok R S 1995b J. Polym. Mater. 1223

Khare P K and Jain S K 1997 Indian J. Pure \& Appl. Phys. 35 408

Khare P K, Sandeep Shrivastava and Srivastava A P 1994 Indian J. Phys. A68 129

Khare P K, Keller J M, Gaur M S, Singh Ranjeet and Datt S C 1996 Polym. Int. 36303

Khare P K, Jain S K and Paliwal S K 1997a Bull. Mater. Sci. 20 699

Khare P K, Kuraria R K and Jain S K 1997b Bull. Mater. Sci. 14133

Khare P K, Paliwal S K, Kuraria R K, Vishwakarma H L, Ashish Verma and Jain S K 1998a Bull. Mater. Sci. 21139

Khare P K, Ashish Verma and Paliwal S K 1998b Bull. Mater. Sci. 21207

Kosaki M, Yoda M and Ieda M 1971 J. Phys. Soc. Jap. 311598

Kulshrestha Y K and Srivastava A P 1980 Thin Solid Films 69 269

Lamb D R 1967 Electronic conduction mechanism in thin insulating films (London: Methuen \& Co.)

Lampert M A and Mark P 1970 Current injection in solids (NY: Academic Press) p. 21

LaxmiNarayana K, Dasardhudu and Rao N 1994 Polym. Int. 35 315

Marikhin V A, Shitsker A I and Yastrebinskii A A 1965 Sov. Phys. Solid State 7352

Mark H F, Gaylord N G and Bikalas N M (eds) 1965 Encyclopedia of polymer science and technology (NY: Interscience)

Ranjeet Singh, Khare P K and Reeta Singh 1997 Indian J. Phys. A71 39

Shrivastava S K, Ranade J D and Srivastava A P 1979 Jpn J. Appl. Phys. 182303

Simmons J G 1970 Handbook of thin film technology (NY: McGraw Hill)

Sinha H C, Talwar I M and Srivastava A P 1981 Thin Solid Films 82229

Singh Ranjeet and Srivastava A P 1972 Indian J. Pure \& Appl. Phys. 10811

Upadhyay J K, Khare P K, Ashish Verma and Paliwal S K 1998 J. Polym. Mater. 15289

Wintle H J 1971 Jpn J. Appl. Phys. 10659 